\title{
Combination of TRAIL and actinomycin D liposomes enhances antitumor effect in non-small cell lung cancer
}

This article was published in the following Dove Press journal:

International Journal of Nanomedicine

14 March 2012

Number of times this article has been viewed

\section{Liangran Guo 1,2,4 \\ Li Fan ${ }^{1,2}$ \\ Jinfeng Ren ${ }^{1,2}$ \\ Zhiqing Pang ${ }^{1,2}$ \\ Yulong Ren ${ }^{1,2}$ \\ Jingwei $\mathrm{Li}^{1,2}$ \\ Ziyi Wen ${ }^{1,3}$ \\ Yong Qian ${ }^{1,2}$ \\ Lin Zhang ${ }^{1,2}$ \\ Hang $\mathrm{Ma}^{4}$ \\ Xinguo Jiang ${ }^{1,2}$}

'School of Pharmacy, Fudan University, Zhangheng Road, Shanghai, ${ }^{2}$ Key

Laboratory of Smart Drug Delivery,

Ministry of Education and PLA,

Shanghai, ${ }^{3}$ School of Pharmacy,

Shenyang Pharmaceutical University,

Shenyang, People's Republic of China;

${ }^{4}$ College of Pharmacy, University of

Rhode Island, RI, USA
Correspondence: Xinguo Jiang

School of Pharmacy, Fudan University,

\#826, Zhangheng Rd, Shanghai 201203,

People's Republic of China

Tel +862151980067

Fax +862151980069

Email xgjiang@shmu.edu.cn
Abstract: The intractability of non-small cell lung cancer (NSCLC) to multimodality treatments plays a large part in its extremely poor prognosis. Tumor necrosis factor-related apoptosisinducing ligand (TRAIL) is a promising cytokine for selective induction of apoptosis in cancer cells; however, many NSCLC cell lines are resistant to TRAIL-induced apoptosis. The therapeutic effect can be restored by treatments combining TRAIL with chemotherapeutic agents. Actinomycin D (ActD) can sensitize NSCLC cells to TRAIL-induced apoptosis by upregulation of death receptor 4 (DR4) or 5 (DR5). However, the use of ActD has significant drawbacks due to the side effects that result from its nonspecific biodistribution in vivo. In addition, the short half-life of TRAIL in serum also limits the antitumor effect of treatments combining TRAIL and ActD. In this study, we designed a combination treatment of long-circulating TRAIL liposomes and ActD liposomes with the aim of resolving these problems. The combination of TRAIL liposomes and ActD liposomes had a synergistic cytotoxic effect against A-549 cells. The mechanism behind this combination treatment includes both increased expression of DR5 and caspase activation. Moreover, systemic administration of the combination of TRAIL liposomes and ActD liposomes suppressed both tumor formation and growth of established subcutaneous NSCLC xenografts in nude mice, inducing apoptosis without causing significant general toxicity. These results provide preclinical proof-of-principle for a novel therapeutic strategy in which TRAIL liposomes are safely combined with ActD liposomes.

Keywords: TRAIL, actinomycin D, liposomes, combination treatment, non-small cell lung cancer

\section{Introduction}

Non-small cell lung cancer (NSCLC) accounts for approximately $85 \%$ of all cases of lung cancer, which is the leading cause of cancer-related mortality in both men and women throughout the world. ${ }^{1}$ Systemic chemotherapy has been considered the main treatment for patients with early-stage NSCLC and part of the multimodal therapeutic strategy for advanced-stage NSCLC. ${ }^{1,2}$ However, limited efficiency has been achieved using chemotherapy. For example, the use of cytotoxic chemotherapy is associated with a response rate of $20 \%-35 \%$ and a median survival time of $10-12$ months in patients with advanced NSCLC. ${ }^{3}$ This may be due to multidrug resistance in NSCLC. Several resistance proteins, including P-glycoprotein, lung resistance protein, and multidrug resistance-associated protein, have been proven to be simultaneously involved in multidrug resistance of NSCLC. ${ }^{4}$ Therefore, there is a need to develop new anticancer drugs and novel regimens capable of killing drug-resistant cells. Activation of the death receptor pathway may provide a new modality in NSCLC treatment because of the 
ability of death receptors to induce apoptosis directly, thus bypassing cellular drug resistance. ${ }^{5}$

A new candidate, tumor necrosis factor-related apoptosisinducing ligand (TRAIL), was identified as a powerful activator of programmed cell death or apoptosis in tumor cells that also spared normal cells. ${ }^{6}$ TRAIL-induced apoptosis starts with the binding of TRAIL to its cognate receptors DR4/5. This binding leads to the formation of a death-inducing signaling complex and subsequent activation of caspase- 8 . Caspase- 8 then activates downstream effector caspases, such as caspase-3 and caspase-6, ultimately resulting in apoptosis. ${ }^{7}$ The selectivity of TRAIL for tumor cells has greatly spurred research to explore its potential as an anticancer therapy. ${ }^{6,89}$ Although TRAIL is capable of inducing apoptosis in tumor cells of diverse origin, recent studies have shown that the majority of NSCLC lines, such as A-549 and H-522, are resistant to the apoptotic effects of TRAIL, suggesting that the use of TRAIL alone may be ineffective for NSCLC therapy. ${ }^{10}$

There is increasing evidence that conventional chemotherapeutic agents upregulate death receptors in human cancer cells. Therefore, it is possible that these conventional chemotherapeutic drugs would enhance the cytotoxicity of TRAIL. This approach might be useful in killing drugresistant cells. Importantly, a number of studies have shown that treatments combining TRAIL with chemotherapy have synergistic effects in several human tumor types and can overcome resistance to either of the agents. ${ }^{10}$ Actinomycin D (ActD), which is known to be a potent cytotoxic drug, can sensitize pancreatic cancer and NSCLC cells to TRAILinduced apoptosis. ${ }^{11-13}$ However, the effectiveness of this combination therapy on NSCLC xenograft model systems has not been reported. Therefore, evaluation of the therapeutic efficiency of combined ActD/TRAIL treatment on NSCLC xenografts is necessary and feasible.

However, use of ActD has inevitable drawbacks due to the side effects that result from its nonspecific biodistribution in vivo. In addition, the short half-life of TRAIL in serum ${ }^{14}$ could also limit the antitumor effect of combined treatment with TRAIL and ActD. In addition, some NSCLC cell lines demonstrate cross-resistance to both TRAIL and ActD, making combination therapies less effective. ${ }^{4,10}$

In recent years, liposomes have garnered attention for cancer therapy because of their biocompatibility and versatility of formulation. ${ }^{15}$ Compared with free antitumor drugs, drug-loaded long-circulating liposomes show a long circulation time in plasma, enhanced accumulation in tumor tissues, and superior therapeutic activity. Our previous studies have indicated that the combination of TRAIL liposomes and doxorubicin liposomes enhances antitumor effects. ${ }^{16,17}$ In spite of these potential advantages, long-circulating liposomes have not been tested as a carrier for ActD, and the clinical application of TRAIL liposomes combined with ActD liposomes may result in enhanced antitumor effects.

The objective of the present study was to determine whether ActD liposomes augment TRAIL liposome-induced apoptosis of A-549, a NSCLC cell line, and to investigate the underlying mechanisms by which this combined treatment achieves enhanced elimination of cancer cells. We used the A-549 cell line as a multidrug resistant lung tumor cell model because of the presence of ATP-binding cassette efflux pumps such as P-glycoprotein, multidrug resistanceassociated protein, and breast cancer resistance protein in A-549 cells. ${ }^{18}$

\section{Materials and methods Materials and animals}

ActD was obtained from Life Science Co (Shanghai, China). Human recombinant TRAIL was supplied by the Shanghai Qiaer Biotechnology Co, Ltd, (Shanghai, China). 2-(4-Amidinophenyl)-6-indolecarbamidine dihydrochloride (DAPI) and MTT were purchased from Sigma (St Louis, MO). Antihuman death receptor 4 (DR4) and 5 (DR5) antibodies, human recombinant DR5/Fc and DR4/Fc chimera proteins, monoclonal antihuman TRAILR1-phycoerytherin, and monoclonal antihuman TRAILR2-phycoerytherin were obtained from R \& D Systems Inc (Minneapolis, MN). Antibodies to caspase-9, caspase-3, and caspase- 8 were obtained from Bioworld Technology Inc (Louis Park, MN). Egg yolk phosphatidylcholine (EPC) was purchased from QP Corporation (Tokyo, Japan). Cholesterol (CHOL) and 1,2distearoyl-sn-glycero-3-phosphoethanolamine-n-[methoxy (polyethylene glycol)-2000] (DSPE-mPEG-2000) were obtained from Avanti Polar Lipids Inc (Alabaster, AL). Fetal bovine serum and Roswell Park Memorial Institute (RPMI) 1640 were ordered from Gibco (Carlsbad, CA). Doubledistilled water was purified using a Millipore Simplicity System (Millipore, Bedford, MA). All other materials were of analytical grade and used without further purification.

The NSCLC cell line A-549 was obtained from the Institute of Biochemistry and Cell Biology at the Shanghai Institutes for Biological Sciences of the Chinese Academy of Sciences (Shanghai, China). The cells were cultured in RPMI 1640 supplemented with $100 \mathrm{U} / \mathrm{mL}$ penicillin, $100 \mu \mathrm{g} / \mathrm{mL}$ streptomycin, and $10 \%$ fetal bovine serum at $37^{\circ} \mathrm{C}$ in a humidified $5 \% \mathrm{CO}_{2}$ atmosphere. 
BALB/c mice (BK Lab Animal Co Ltd, Shanghai, China) were treated according to protocols approved by the Ethics Committee of Fudan University.

\section{TRAIL stability study}

TRAIL was dissolved in phosphate-buffered solution (pH 7.4) at a concentration of $100 \mu \mathrm{g} / \mathrm{mL}$, and then $3 \mathrm{~mL}$ of $100 \mu \mathrm{g} / \mathrm{mL}$ TRAIL was divided into samples $1(1 \mathrm{~mL})$, $2(1 \mathrm{~mL})$, and $3(1 \mathrm{~mL})$. Sample 1 was kept at room temperature as a control. Sample 2 was incubated in a water bath at $40^{\circ} \mathrm{C}$ for one hour, while sample 3 was kept at $50^{\circ} \mathrm{C}$ for one hour. The cytotoxicity of each sample on A-549 cells was then investigated using the MTT assay.

\section{Preparation of ActD liposomes}

The formulation of ActD liposomes was as follows: EPC:CHOL:DSPE-mPEG-2000:ActD = 20:5:5:0.8 (w/w). Briefly, ActD $(0.8 \mathrm{mg})$ and lipids with the compositions described above were dissolved in $6 \mathrm{~mL}$ of chloroform. Following the addition of $4 \mathrm{~mL}$ of phosphate-buffered solution (pH 7.4), a dry lipid film prepared from the mixture was vortexed for 5 minutes and then suspended for 4 hours. The liposomal suspension was then subjected to a sonication procedure of $200 \mathrm{~W}$ for 20 seconds (intermittent). Large liposomes and nonentrapped ActD particles were removed by three passes through polycarbonate membrane filters with $0.22 \mu \mathrm{m}$ pores. The ActD liposomes were stored at $4^{\circ} \mathrm{C}$ until use.

The TRAIL liposomes were prepared as previously reported. ${ }^{17}$ The lipid composition of the TRAIL liposomes was as follows: EPC:CHOL:DSPE-mPEG-2000 = 65:15:15 $(w / w)$. Briefly, lipids with the compositions described above (total weight $95 \mathrm{mg}$ ) were dissolved in chloroform, dried into a thin film in a round bottom flask on a rotary evaporator under reduced pressure at $40^{\circ} \mathrm{C}$. The dried lipid film was rehydrated in $4 \mathrm{~mL}$ of $5 \mathrm{mg} / \mathrm{ml}$ TRAIL solution adjusted to pH 7.4 and 290 osmolarity with $\mathrm{NaCl}$ at room temperature, sonicated in an ice bath, and then subsequently extruded three times through a $0.22 \mu \mathrm{m}$ pore size polycarbonate filter. The resulting liposomes were filtered through sepharose (CL-4B) to remove nonentrapped TRAIL.

\section{Characterization of liposomes}

Liposome size was determined by dynamic light scattering using a zeta potential/particle sizer Nicomp 380 ZLS (Particle Sizing Systems, Santa Barbara, CA). In addition, liposomes were observed using transmission electron microscopy (H-600, Hitachi, Japan) following negative staining with 1\% sodium phosphotungstate solution for 2 minutes.
The concentration of ActD within the liposomes was measured by ultraviolet absorbance at $458 \mathrm{~nm}$ (UV-2401PC, Shimadzu, Tokyo, Japan). Briefly, $2 \mathrm{~mL}$ of the filtered ActD liposomes or nonfiltered ActD liposomes was dissolved in $7 \mathrm{~mL}$ ethanol, and the final volume was adjusted to $10 \mathrm{~mL}$ with ethanol. After vortexing for 10 minutes, this suspension was filtered with polycarbonate membrane filters with $0.22 \mu \mathrm{m}$ pores to remove undissolved materials. The ultraviolet absorbance of each sample was then measured and the concentration of ActD was determined accordingly. The loading efficiency was calculated using the following equation:

$$
\text { Loading efficiency }(\%)=\mathrm{Fi} / \mathrm{Ft} \times 100
$$

where Fi is the concentration of ActD loaded in the liposomes after their dissolution in ethanol and $\mathrm{Ft}$ is the initial concentration of ActD.

In contrast, nonentrapped TRAIL was tested using the Coomassie Brilliant Blue G-250 binding assay. ${ }^{19}$ Briefly, the nonentrapped TRAIL was collected by passing TRAIL liposomes through sepharose; $2.5 \mathrm{~mL}$ of Coomassie Brilliant Blue G-250 $(0.5 \mathrm{mg} / \mathrm{mL})$ was then added to $1 \mathrm{~mL}$ of the collected TRAIL solution. A series of concentrations of TRAIL $(2-80 \mu \mathrm{g} / \mathrm{mL})$ was used as a control. The ultraviolet $\mathrm{A}_{450}$ and $\mathrm{A}_{590}$ of each sample were measured within 20 minutes. The concentration of TRAIL was determined by $\mathrm{A}_{590} / \mathrm{A}_{450}$. The loading efficiency of TRAIL was calculated using the following equation:

$$
\text { Loading efficiency }(\%)=(\mathrm{Ft}-\mathrm{Fi}) / \mathrm{Ft} \times 100
$$

where Fi is the concentration of nonentrapped TRAIL after the liposomes were passed through the sepharose column and Ft is the initial concentration of TRAIL.

The release of TRAIL or ActD from liposomes was examined by incubating $1 \mathrm{~mL}$ of liposomes in a dialysis bag immersed in $20 \mathrm{~mL}$ of phosphate-buffered solution ( $\mathrm{pH}$ 7.4). The entire system was stirred at $37^{\circ} \mathrm{C}$. The amount of TRAIL and ActD released at each time point was determined using Coomassie Brilliant Blue G-250 binding and ultraviolet analysis, respectively.

\section{Nuclear staining}

A-549 cells were placed in six-well culture plates $\left(5 \times 10^{5} /\right.$ well $)$ and incubated for about 12 hours. The cells were then treated for 12 hours with TRAIL liposomes alone ( $100 \mathrm{ng} / \mathrm{mL})$, ActD liposomes alone $(1 \mu \mathrm{g} / \mathrm{mL})$ or both. The culture medium 
was then removed and cells were washed with phosphatebuffered solution twice and fixed with $3.7 \%$ paraformaldehyde in phosphate-buffered solution for 10 minutes at room temperature. Fixed cells were washed three times with phosphate-buffered solution and stained with $0.5 \mu \mathrm{g} / \mathrm{mL}$ DAPI solution for 15 minutes in the dark at room temperature. Finally, cells were observed via fluorescence microscopy (Leitz, Germany).

\section{Cytotoxicity assay}

Cytotoxicity was evaluated using the MTT survival assay. Briefly, A-549 cells were plated at 10,000 cells/well in 96-well microtiter plates overnight. Cells were then treated with $100 \mu \mathrm{L}$ fresh medium containing ActD liposomes alone, TRAIL liposomes alone, or both agents and cultured for 24 hours. Following an additional 4 hours of culture with $0.5 \mathrm{mg} / \mathrm{mL}$ MTT, samples were analyzed using a microplate reader (Multiskan MK3, Thermo Scientific, MA). The effects of the treatments were expressed as a percentage of growth inhibition using untreated cells as the noninhibited control.

\section{Western blot analysis}

Preparation of whole cell protein lysates and Western blot analysis were performed as previously described. ${ }^{20,21}$ In brief, whole cell protein lysates were electrophoresed through denaturing polyacrylamide slab gels, and the protein bands were transferred to a polyvinylidene fluoride membrane. The membrane was then probed with primary antibodies to caspase-9 (1:1000), caspase-8 (1:1000), caspase-3 (1:1000), DR5 (1:1000), DR4 (1:1000), and GAPDH (1:1000) from Sigma, St Louis, MO. Primary antibodies were detected using goat antimouse or antirabbit horseradish peroxidase-link secondary antibodies (1:10,000). After washing, the ECL chemical reagents were added to the membrane and chemiluminescence was detected by exposure of the membranes to Kodak-X-Omat films for 30 seconds to 10 minutes.

\section{Membrane expression of DR4 and DR5}

Cells were analyzed for surface expression of DR4 and DR5 by direct staining with monoclonal antihuman TRAILR1phycoerytherin and monoclonal anti-human TRAILR2phycoerytherin. Briefly, cells were seeded at $1 \times 10^{5}$ cells/ well in 24-well plates overnight. Cells were then treated with fresh medium containing ActD liposomes and further cultured for 8 hours. Cells were stained with $0.5 \mathrm{~mL}$ phosphate-buffered solution containing $1 \mu \mathrm{g} / \mathrm{mL}$ of monoclonal antihuman TRAILR1-phycoerytherin or monoclonal antihuman TRAILR2-phycoerytherin antibody at $4^{\circ} \mathrm{C}$ for
30-45 minutes. After washing with phosphate-buffered solution, the expression of DR4 or DR5 was analyzed using fluorescence microscopy (Leitz, Germany).

\section{Antitumor activity of ActD and TRAIL liposome combination treatment}

A-549 cells $\left(2 \times 10^{6}\right)$ were suspended in $0.1 \mathrm{~mL}$ phosphatebuffered solution and injected subcutaneously into the right flank of BALB-C strain female nude mice 6-8 weeks of age. For the treatment of the established xenografts, the tumors were permitted to establish and grow for 20 days (tumor volume, 50-100 $\mathrm{mm}^{3}$ ). After tumor formation, the mice were divided into seven treatment groups (five mice/group) and received injection of the following treatments via tail vein: control ( $\mathrm{pH} 7.4$ phosphate-buffered solution); TRAIL $0.6 \mathrm{mg} / \mathrm{kg}$; ActD $0.06 \mathrm{mg} / \mathrm{kg}$; TRAIL $0.6 \mathrm{mg} / \mathrm{kg}+$ ActD $0.06 \mathrm{mg} / \mathrm{kg}, \mathrm{pH} 7.4$ phosphate-buffered solution; TRAIL liposomes $0.6 \mathrm{mg} / \mathrm{kg}$; ActD liposomes $0.06 \mathrm{mg} / \mathrm{kg}$; TRAIL liposomes $0.6 \mathrm{mg} / \mathrm{kg}+$ ActD liposomes $0.06 \mathrm{mg} / \mathrm{kg}$. The administration of drugs was carried out daily for 12 days. Tumor size was measured with calipers every day. Tumor volume was calculated using the following formula: $1 / 2 \times$ (larger diameter $) \times($ smaller diameter $){ }^{2}$ Systemic toxicity of the treatments was assessed by changes in mouse body weight.

\section{Statistical analysis}

Statistical evaluations of data were performed using the unpaired Student's $t$-test and one-way analysis of variance. Data were expressed as the mean \pm standard deviation. $P<0.05$ was considered to be significant.

\section{Results}

\section{TRAIL stability study}

Although the sonication of liposomes was performed in an ice bath, it is difficult to avoid heat buildup in a liposomal suspension. Because the activity of bioactive proteins can be easily damaged by heat, we tested the susceptibility of TRAIL to heat. As shown in Figure 1, heat treatment of TRAIL at $40^{\circ} \mathrm{C}$ or $50^{\circ} \mathrm{C}$ for one hour had only a slight influence on TRAIL bioactivity, suggesting that the bioactivity of TRAIL may not be damaged by the sonication. In addition, the sonication procedure did not significantly damage the TRAIL potent (data not shown). These results indicated that the sonication procedure can be utilized in the preparation of TRAIL liposomes. To the best of our knowledge, a stability study of this type has not been previously reported. 


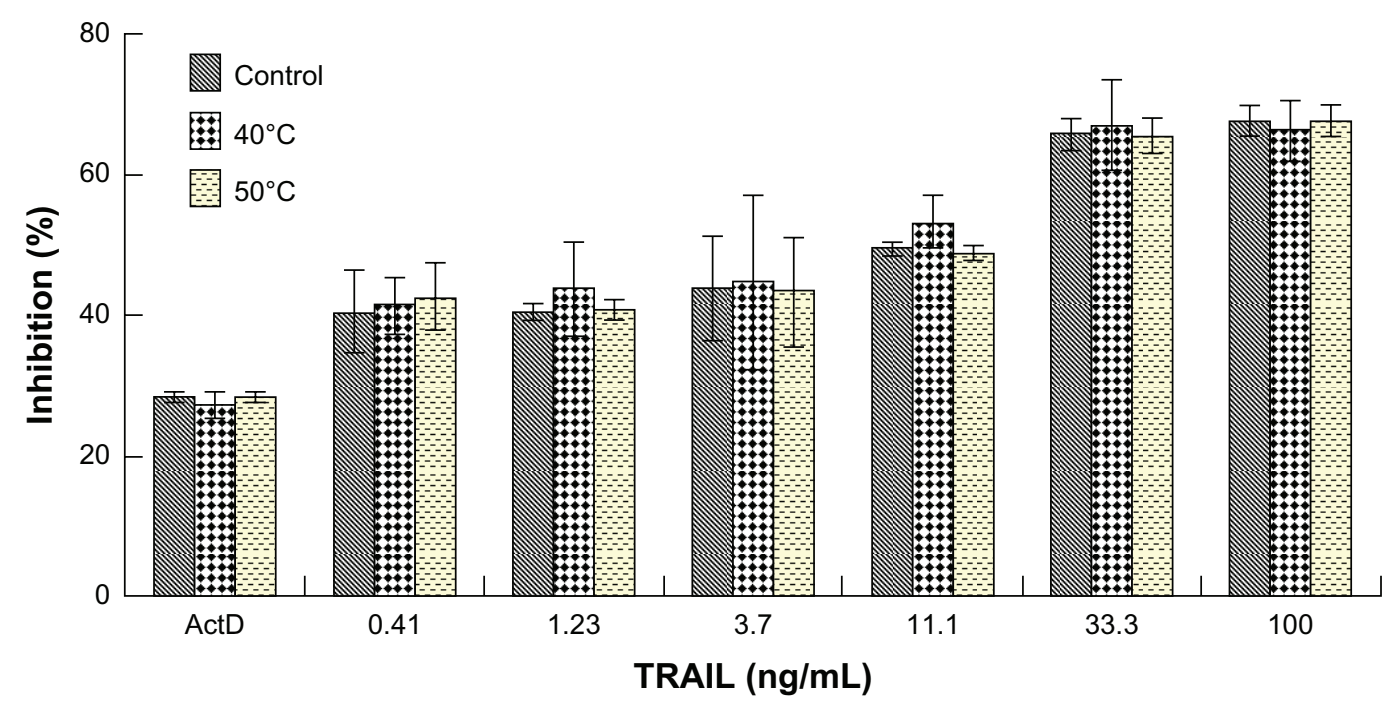

Figure I Effect of heat treatment on cytotoxicity of TRAIL to A-549 cells. Because A-549 cells are resistant to TRAIL, we added I $\mu g / m L$ of actinomycin D to each concentration of TRAIL as a sensitizer.

Abbreviation: TRAIL, tumor necrosis factor-related apoptosis-inducing ligand.

\section{Characteristics of liposomes}

The physical properties of liposomes are listed in Table 1. Dynamic light scattering results demonstrated that volumebased diameters of TRAIL liposomes and ActD liposomes were around $110 \mathrm{~nm}$, which is especially suitable for accumulation in tumor tissue due to enhanced permeation and retention. ${ }^{22}$ Importantly, the in vitro release results for the TRAIL liposomes and ActD liposomes showed that the liposomes can sustain ActD and TRAIL release, suggesting the possibility of a prolonged circulation time for both drugs. We also examined the shape and size of these liposomes under a transmission electron microscope (Figure 2). Most TRAIL and ActD liposomes were spherical and had a regular shape.

\section{Cytotoxicity assay}

For qualitative assessment of apoptosis induced by TRAIL liposomes alone, ActD liposomes alone, or both agents, we analyzed chromatin condensation and apoptotic bodies. As shown in Figure 3A, treatment with TRAIL liposomes $(100 \mathrm{ng} / \mathrm{mL})$ alone for 12 hours did not induce any morphological features or apoptotic bodies indicative of cell

Table I Physical properties of the liposomal formulations

\begin{tabular}{lcc}
\hline & TRAIL liposomes & ActD liposomes \\
\hline Loading efficiency (\%) & $10.4 \pm 3.9$ & $92.0 \pm 4.5 \mathrm{I}$ \\
Particle size (nm) & $115.6 \pm 25.4$ & $110.5 \pm 45.3$ \\
Cumulative release & $71.2 \pm 4.3$ & $62.9 \pm 5.7$ \\
at 24 hours (\%) & & \\
\hline
\end{tabular}

Abbreviations: TRAIL, tumor necrosis factor-related apoptosis-inducing ligand; ActD, actinomycin D. death. Treatment with ActD liposomes $(1.0 \mu \mathrm{g} / \mathrm{mL})$ alone induced only a slight increase in such morphological changes and apoptotic bodies. In contrast, after combined treatment with TRAIL liposomes and ActD liposomes, appearance of apoptotic bodies was observed in A-549 cells. After washing with phosphate-buffered solution and staining with DAPI, the presence of apoptotic bodies and remarkably lower growth density in cells treated with the combination treatment was apparent (Figure 3B). This indicates that numerous dead tumor cells produced by the combination treatment were washed away by phosphate-buffered solution. These results demonstrate that ActD liposomes significantly enhance apoptosis induced by TRAIL liposomes in A-549 cells.

To investigate further the cytotoxic effects of TRAIL liposomes and/or ActD liposomes, we treated A-549 cells with the indicated agents and subjected them to the MTT assay.
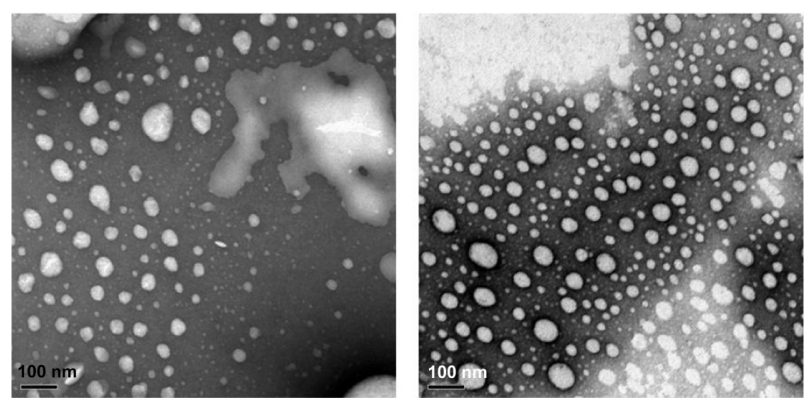

Figure 2 Transmission electron micrographs of liposomes. TRAIL liposomes (left), ActD liposomes (right).

Note: $B a r=100 \mathrm{~nm}$.

Abbreviations: TRAIL, tumor necrosis factor-related apoptosis-inducing ligand; ActD, actinomycin D. 


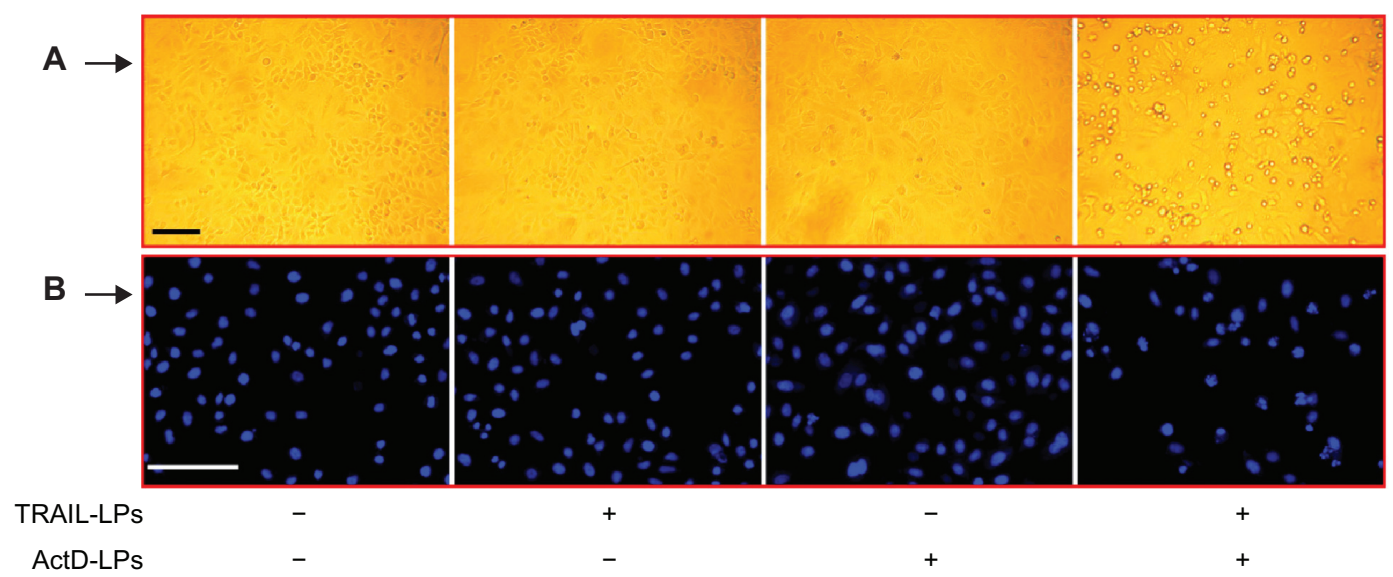

Figure 3 Induction of apoptosis in A-549 cells by TRAlL liposomes and/or ActD liposomes. (A) Bar $=250 \mu \mathrm{m}$. (B) Bar $=250 \mu \mathrm{m}$. Abbreviations: TRAIL, tumor necrosis factor-related apoptosis-inducing ligand; ActD, actinomycin D; LPs, liposomes.

As shown in Figure 4A, neither TRAIL liposomes nor ActD liposomes can significantly inhibit cell growth as single agents; however, combined treatment with TRAIL liposomes and ActD liposomes resulted in a sharp increase in cell inhibition. This result is consistent with results reported elsewhere. ${ }^{10}$ Importantly, when A-549 cells were treated with TRAIL liposomes $(1.4-1000 \mathrm{ng} / \mathrm{mL})$ and ActD $(1.4-1000 \mathrm{ng} / \mathrm{mL})$ at a fixed ratio $(1: 1, \mathrm{w} / \mathrm{w})$ for 24 hours, the inhibition of cells significantly increased compared with single treatment at each concentration point, confirming that
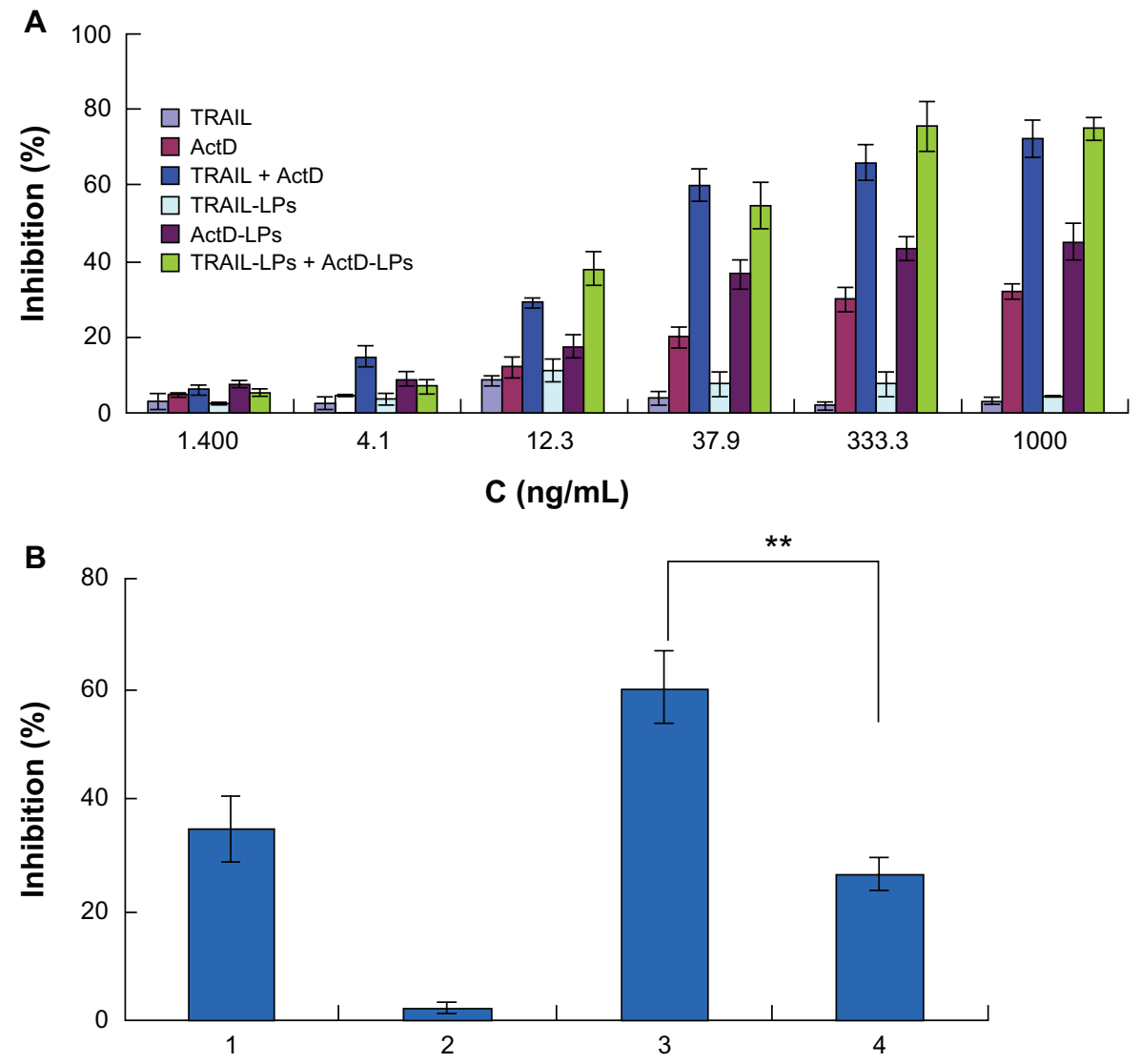

Figure 4 (A) Effect of TRAIL liposomes and/or ActD liposomes on growth inhibition of A-549 cells. (B) Cell inhibition induced by forward or reverse sequential treatment of TRAIL liposomes and ActD liposomes: I, ActD liposome treatment for 24 hours; 2, TRAIL liposome treatment for 24 hours; 3 , ActD liposome treatment for 8 hours $\rightarrow$ TRAIL liposome treatment for 16 hours; 4, TRAIL liposome treatment for 16 hours $\rightarrow$ ActD liposome treatment for 8 hours.

Note: $* * P<0.01$

Abbreviations: TRAIL, tumor necrosis factor-related apoptosis-inducing ligand; ActD, actinomycin D; LPs, liposomes. 
cotreatment with TRAIL liposomes and ActD liposomes is effective in inducing cell death. Similar results were also found in the combined treatment with free TRAIL and ActD for 24 hours in the same cell line.

Because A-549 cells are highly resistant to TRAILinduced apoptosis, we next sought to test whether A-549 cells can be inhibited by pretreatment with ActD liposomes before treatment with TRAIL liposomes. Interestingly, if A-549 cells were pretreated with ActD liposomes alone for 8 hours followed by treatment with TRAIL liposomes for an additional 16 hours, effective cell inhibition was also observed (Figure 4B). To examine this synergistic interaction, the reverse sequence of drug exposure was used, where cells were pretreated with TRAIL liposomes for 16 hours, followed by treatment with ActD liposomes for an additional 8 hours. This reverse sequence of drug exposure resulted in significantly less cell inhibition than the sequential treatment of cells with ActD liposomes followed by TRAIL liposomes. Taken together, these results indicate that sequential treatment with ActD liposomes followed by TRAIL liposomes or synchronous treatment of ActD liposomes and TRAIL liposomes can be used to kill A-549 cells.

\section{ActD liposomes induce upregulation of TRAIL receptor DR4 and DR5}

It has been previously established that TRAIL induces apoptosis through binding to cell receptors. Several TRAIL receptors including DR4, DR5, decoy receptor 1 (DcR1), 2 (DcR2), and osteoprotegerin have been identified to date. Both DR4 and DR5 contain a conserved death domain motif and transduce TRAIL-mediated death signals to the intracellular apoptotic machinery. Unlike DR4 and DR5, DcR1 lacks an intracellular domain, while DcR2 has a truncated death domain. Thus, these two receptors act as decoy receptors that antagonize TRAIL-induced apoptosis by competing for ligand binding. ${ }^{23}$ Several mechanisms accounting for the blocking of TRAIL-induced apoptosis have been reported in individual cancer cell lines, including the simultaneous expression of decoy receptors, ${ }^{24}$ overexpression of antiapoptotic molecules, ${ }^{25}$ and loss of caspase- 8 expression. ${ }^{26}$ A number of studies have shown that anticancer drugs can induce DR5 to sensitize cancer cells to TRAIL. ${ }^{27-30}$ It has also been shown that DR4 can be transcriptionally induced by chemotherapeutic drugs. ${ }^{31}$ However, another study showed that sensitization can occur without increased TRAIL receptor expression. ${ }^{32}$ Regardless of total DR4 and DR5 protein levels, their absence on the cell surface is sufficient to account for the failure to form a TRAIL death-inducing signaling complex and later steps in apoptosis.

In light of the studies cited above, it is important to know whether ActD liposome treatment leads to regulation of DR4 and DR5 expression on the cell surface. We therefore evaluated TRAIL receptor expression on the surface of A-549 cells at 4, 8, and 12 hours after treatment with ActD liposomes. Western blot analysis showed that the basal level of expression of both DR4 and DR5 increased significantly after treatment with ActD liposomes (Figure 5A). The increased expression of DR4 and DR5 in A-549 cells may be at least one of the reasons for the enhanced cytotoxicity of TRAIL liposomes and ActD liposome combination treatment.

A

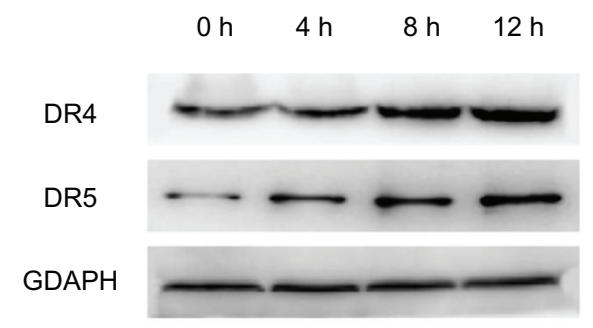

B
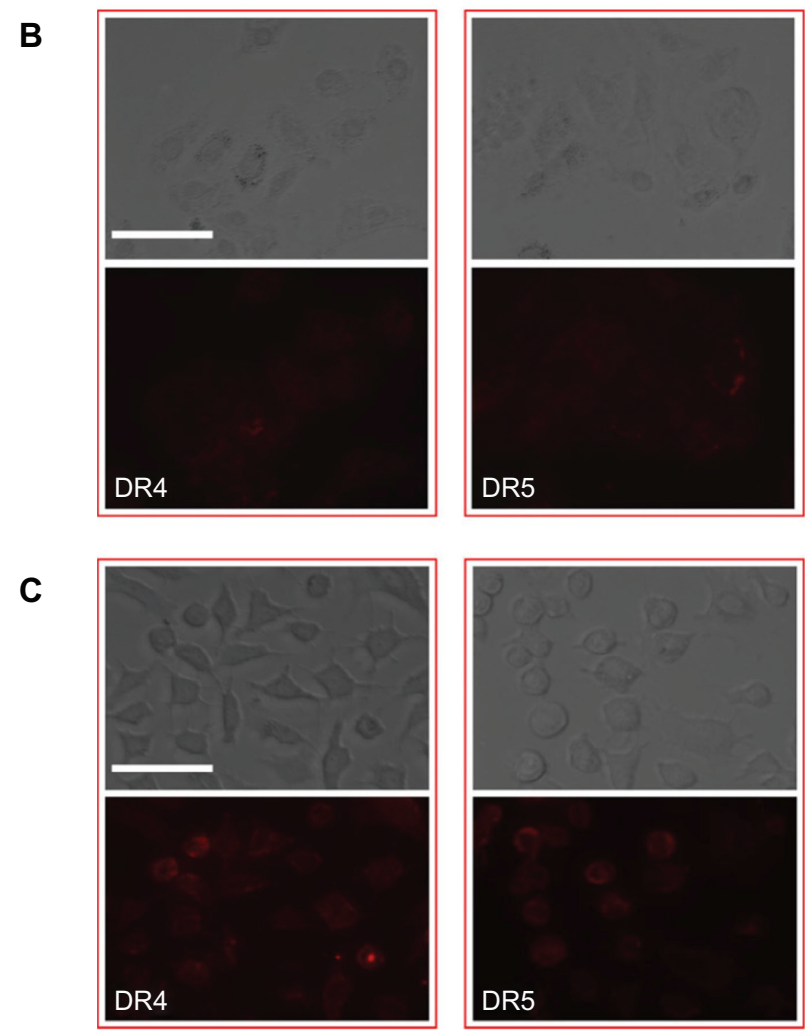

Figure 5 (A) Effects of actinomycin D liposomes on DR4 and DR5 expression. A-549 cells were treated with actinomycin D liposomes $(0.125 \mu \mathrm{g} / \mathrm{mL})$ for 4 , 8, or 12 hours, respectively. Cell lysates were examined by Western blot. (B) Fluorescence micrographs of DR4 and DR5 expression on untreated A-549 cells. (C) Fluorescence micrographs of increased DR4 and DR5 expression on A-549 cells induced by actinomycin $\mathrm{D}$ liposomes.

Note: $\mathrm{Bar}=125 \mu \mathrm{m}$. 
The DR4 and DR5 upregulation induced by ActD liposomes and subsequent enhancement of TRAIL-mediated cytotoxicity in A-549 cells may correlate with p53 status. p53 acts as a transcription factor and induces apoptosis by increasing gene expression of DR4 and DR5, resulting in upregulation of DR4 and DR5 protein synthesis..$^{28,33,34}$ Cells treated with ActD liposomes expressed higher levels of both DR4 and DR5 compared with untreated cells (Figure 5B and C). In addition, the DR4 and DR5 were distributed not only on the cell surface but also in the cytoplasm. It is possible that ActD liposomes induce the redistribution of DR4 and DR5 to lipid rafts at the plasma membrane, favoring their aggregation. ${ }^{35}$ Although mRNA expression of both DR4 and DR5 is present in many cancer cells, there is no correlation between receptor expression levels and the sensitivity of tumors to TRAIL. Recent studies have suggested that the mRNA expression of TRAIL receptors does not necessarily reflect their functional levels, ${ }^{36}$ so we only investigated the expression of DR4 and DR5 proteins in A-549 cells.

\section{Contribution of DR4 and DR5 to apoptotic signaling}

To investigate the relative contribution of DR4 or DR5 to the transduction of the apoptotic signal in sensitive A-549 cells, we selectively blocked each of the receptors with the corresponding chimeras (DR4/Fc and DR5/Fc, respectively). As shown in Figures 6 and 7, only the DR5/Fc chimera abolishes the combined effect of ActD liposomes and TRAIL liposomes, indicating that the sensitization to apoptosis induced by TRAIL liposomes is mediated by one but not both upregulated receptors in A-549 cells.

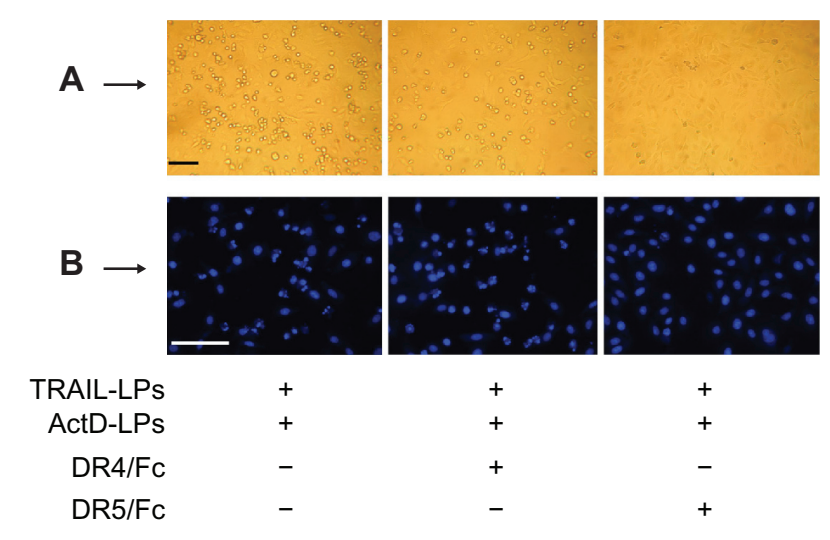

Figure 6 Qualitative assessment of apoptosis induced by TRAIL liposomes, ActD liposomes or both agents. (A) Bar $=250 \mu \mathrm{m}$. (B) Bar $=250 \mu \mathrm{m}$.

Abbreviations: TRAIL, tumor necrosis factor-related apoptosis-inducing ligand; ActD, actinomycin D; LPs, liposomes.

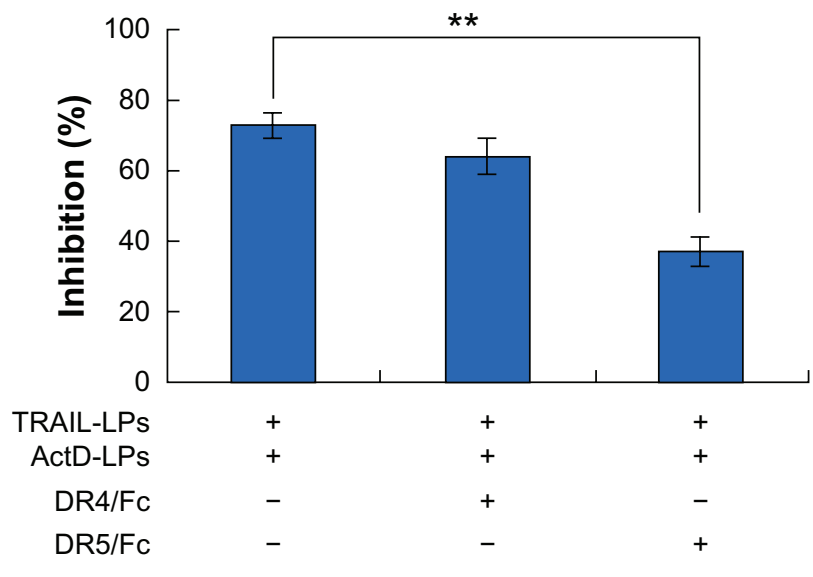

Figure 7 ActD liposomes enhance TRAIL liposome-induced cell inhibition through upregulation of DR5. A-549 cells were treated with ActD liposomes (I $\mu \mathrm{g} / \mathrm{mL})$ and TRAIL liposomes $(100 \mathrm{ng} / \mathrm{mL})$ in the presence or absence of DR4/Fc chimera (1000 ng/mL) or DR5/Fc chimera (1000 ng/mL).

Notes: $* * P<0.01$. Cell inhibition was tested using the MTT assay.

Abbreviations: TRAIL, tumor necrosis factor-related apoptosis-inducing ligand; ActD, actinomycin D; LPs, liposomes.

\section{TRAIL + ActD liposomes enhance cytotoxicity via caspase activation in A-549 cells}

Cell apoptosis is primarily induced by two signaling pathways, the intrinsic mitochondria-mediated pathway and the extrinsic death receptor-induced pathway, and cross-talk between these pathways is mediated by the proapoptotic protein, Bid. ${ }^{29}$ Steps in the intrinsic pathway include cytochrome c release from mitochondria and caspase 9 activation, followed by activation of effector caspases, including caspase 3. Steps in the extrinsic pathway include Fas-associated death domaindependent recruitment and activation of caspase 8 , followed by activation of the same effector caspases involved in the intrinsic pathway. ${ }^{29}$ In previous studies, we determined that DR5 upregulation plays a critical role in the sensitization of A-549 cells mediated by ActD liposomes to TRAIL-induced apoptosis. Next, we examined whether this sensitizing effect was associated with activation of caspase.

Western blot results indicated that TRAIL liposomes induced nearly no cleavage of caspase- 3 or caspase-9, and only slight cleavage of caspase-8 (Figure 8). In contrast, ActD liposomes stimulated detectable processing of caspase-9, whereas the combination treatment induced substantially greater cleavage of caspase-8, caspase- 9 , and caspase-3, therefore resulting in appearance of more active subunits of caspase-3, caspase-8, and caspase-9. These observations suggest that both the intrinsic mitochondria-mediated pathway and the extrinsic death receptor-induced pathway are involved in the combined treatment. 


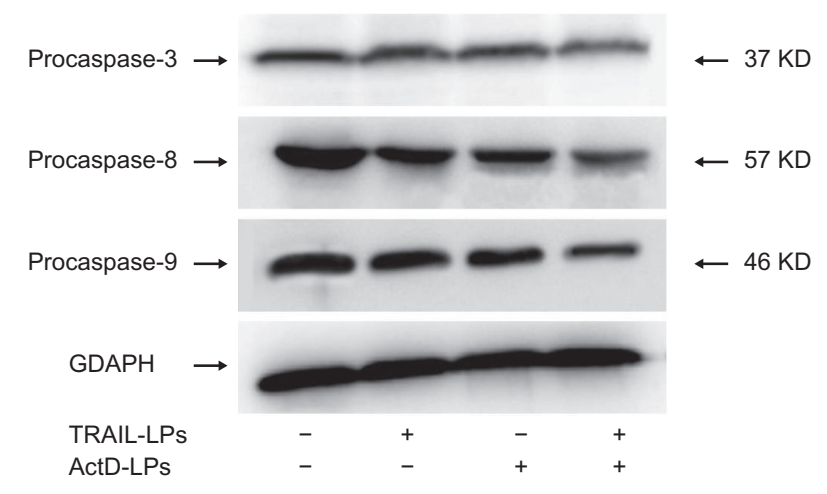

Figure 8 Effects of ActD liposomes or/and TRAIL liposomes on caspase activation A-549 cells were treated with ActD liposomes $(I \mu \mathrm{g} / \mathrm{mL})$ and/or TRAIL liposomes ( $100 \mathrm{ng} / \mathrm{mL})$ for 12 hours.

Abbreviations: TRAIL, tumor necrosis factor-related apoptosis-inducing ligand ActD, actinomycin D; LPs, liposomes.

\section{Treatment of A-549 tumor xenografts with TRAIL + ActD liposomes causes growth suppression}

We next tested the antitumor efficiency of the combination treatment in vivo. Mice bearing subcutaneous A-549 tumor xenografts were treated systemically with indicated agents as described in the Materials and methods sections. The final tumor volume of mice treated with the combination of TRAIL liposomes and ActD liposomes was notably reduced. Furthermore, the tumor growth curve showed that tumor growth was delayed most prominently in the group treated with TRAIL liposomes and ActD liposomes (Figure 9A). The final weight of tumor nodules in the group treated with TRAIL liposomes and ActD liposomes was 392.2 $\pm 119.3 \mathrm{~g}$ (Table 2), which was significantly lower than in the other groups. We were also surprised to find no significant tumor inhibition in the other groups, indicating the superiority of liposomal combination treatment. In this study, body weight change was used as a marker of the safety of the treatment. As shown in Figure 9B, the body weight of mice treated with ActD, ActD liposomes, ActD + TRAIL and ActD liposomes + TRAIL liposomes recovered after cessation of drug treatment. In contrast, the body weight of mice administered phosphate-buffered solution, TRAIL, and TRAIL liposomes gradually increased during the whole treatment period, again demonstrating the minor side effects of TRAIL used alone.

\section{Discussion}

The high frequency of intrinsically TRAIL-resistant NSCLC cell lines requires strategies to sensitize tumors for TRAIL-induced apoptosis. Fortunately, the combined use of TRAIL with chemotherapeutic drugs provides an option for
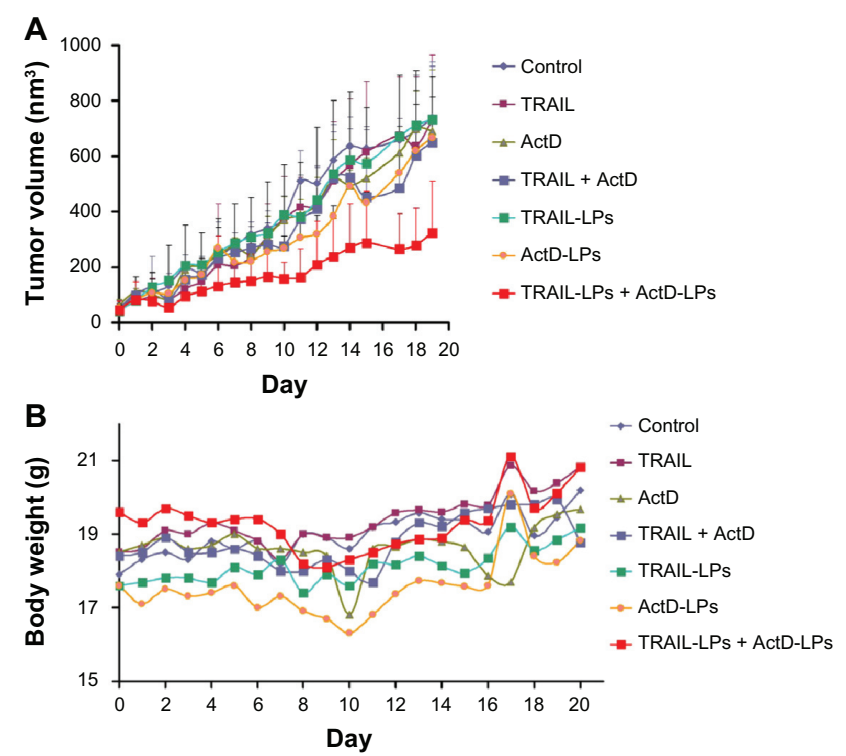

Figure 9 (A) In vivo anticancer efficacy of different treatments in A-549 tumor xenografts and (B) change in body weight as a function of time in subcutaneous A-549 xenograft-bearing mice.

Abbreviations: TRAIL, tumor necrosis factor-related apoptosis-inducing ligand; ActD, actinomycin D; LPs, liposomes.

increasing antitumor activity in NSCLC models. The efficacy of combined treatment with TRAIL and chemotherapy on NSCLC has been demonstrated in a number of studies. ${ }^{10}$ However, the short half-life of TRAIL results in reduced antitumor effects in vivo, which leads to the requirement for larger amounts of TRAIL in clinical trials. Recent studies have reported unanticipated side effects of TRAIL, including proangiogenic-enhanced endothelial cell proliferation, migration, and cytoskeletal reorganization. ${ }^{37,38}$ In addition, a chief concern with conventional chemotherapies is the inevitable side effects from the nonspecific biodistribution of chemotherapeutic drugs. However, to date a strategy to improve on the challenges posed by combination treatment has not been reported in the published scientific literature. Here, we used long-circulating liposomes as carriers to encapsulate ActD and TRAIL with the aim of improving the therapeutic efficiency and reducing the side effects. Our results indicate that the combination of ActD liposomes with TRAIL liposomes has a better therapeutic effect against NSCLC than free TRAIL and ActD combination treatment.

Long-circulating liposomes which are sterically stabilized with surface-coupled polyethylene glycol and have approximately a $100 \mathrm{~nm}$ mean diameter can have an enhanced lifespan in the circulation which is also conferred to their entrapped therapeutic agents. ${ }^{39}$ In recent years, the development of long-circulating liposomes has made it possible to prolong the circulation time of anticancer drugs, target tumors, and 
Table 2 Final tumor weight $(\mathrm{mg})$ of each mouse treated with different formulations

\begin{tabular}{|c|c|c|c|c|c|c|c|c|c|}
\hline & $\mathbf{I}$ & 2 & 3 & 4 & 5 & Average & SD & $\mathbf{P}$ & Inhibition (\%) \\
\hline Control & |37| & 1051 & 842 & 879 & 335 & 895.6 & 376.7 & - & - \\
\hline TRAIL & 1003 & 951 & 940 & 273 & 293 & 692.0 & 374.2 & $>0.05$ & - \\
\hline ActD & 871 & 718 & 800 & 574 & 655 & 723.6 & 116.9 & $>0.05$ & - \\
\hline TRAIL + ActD & 834 & 591 & 778 & 293 & 503 & 599.8 & 218.0 & $>0.05$ & - \\
\hline TRAIL-LPs & 1205 & 984 & 751 & 590 & 381 & 782.2 & 323.4 & $>0.05$ & - \\
\hline ActD-LPs & 687 & 737 & 690 & 227 & 232 & 514.6 & 261.0 & $>0.05$ & - \\
\hline TRAIL-LPs + ActD-LPs & 525 & 423 & 474 & 242 & 297 & 392.2 & 119.3 & $<0.05$ & 56.2 \\
\hline
\end{tabular}

Abbreviations: TRAIL, tumor necrosis factor-related apoptosis-inducing ligand; ActD, actinomycin D; LPs, liposomes; SD, standard deviation.

reduce side effects. For example, doxorubicin is an areaunder-the-curve-dependent anticancer agent and is often used for liposomal targeting to tumors. After encapsulation in longcirculating liposomes, liposomal doxorubicin can be delivered to the extracellular space of tumor tissues. The doxorubicin released in the extracellular space of tumors enhance its effectiveness and substantially increase the therapeutic index. ${ }^{22}$ In this study, the synchronous accumulation of ActD liposomes and TRAIL liposomes in tumors and subsequent drug release in the extracellular space of tumors had a remarkable synergistic effect on the elimination of A-549 cells.

One phenomenon that should be noted is that no significant tumor inhibition was observed in mice treated with the combination of free TRAIL and ActD (Table 2). As reported elsewhere, in order to induce DR4 or DR5 in tumor tissue, chemotherapeutic drugs were usually injected 24 hours prior to administration of TRAIL. ${ }^{5,40,41}$ However, even with the daily systemic injection of the combination of TRAIL and ActD for 12 days, no promising antitumor efficiency was observed in mice. One plausible explanation for this may be that the short half-life of TRAIL allows insufficient time to kill A-549 cells. It is also possible that ActD could not reach a therapeutic concentration in tumor tissues. These results again demonstrate the advantages of liposomes as drug carriers in cancer therapy.

TRAIL seems to require DR5 for maximal killing of A-549 cells. This observation is consistent with previous reports showing that DR5 plays a key role in inducing apoptosis in various types of cancer cells. ${ }^{21,33,42}$ Based on these results, DR5 has been the primary target for development of antibody therapies. There are currently five anti-DR5 antibody products, compared with one anti-DR4 product, in clinical trials. ${ }^{36}$ The potential advantage of the DR5 antibody is specific binding to the target receptor: the antibodies bind selectively and with high affinity to their cognate receptor. ${ }^{43}$ In addition, the half-life of DR5, approximately 18-21 days, is longer than that of TRAIL. However, the wide distribution of DR5 in other tissues and lower accumulation of DR5 at the tumor site limits its efficiency. Therefore, we used liposomes as a carrier to encapsulate TRAIL.

TRAIL-induced apoptosis uses the mitochondriamediated pathway. As we know, cross-talk between the death receptor and mitochondrial pathway is mediated by Bid. The cleavage of Bid activates the proapoptotic members, Bax and Bak, resulting in release of cytochrome $\mathrm{c}$ from the mitochondria. ${ }^{10}$ In contrast, Bcl-2 and Bcl-XL inhibit chemotherapy or TRAIL-induced apoptosis by blocking cytochrome c release. ${ }^{44}$ A-549 cells are resistant to TRAIL, and this may indicate that the activation of caspase- 8 in A-549 cells by TRAIL alone is not sufficient to activate downstream caspase and consequently induce apoptosis. However, when A-549 cells were treated with the combination treatment of ActD liposomes and TRAIL liposomes, mitochondria appear to amplify the apoptotic signals, leading to more activation of caspase-9. Caspase-9, in turn, activates downstream caspases and the cleavage of apoptotic substrates that mediate dismantling of the cell, leading to more cleavage of caspase- 3 into active submits. ${ }^{5}$

In summary, we have developed a strategy that combines ActD liposomes with TRAIL liposomes for the treatment of NSCLC. We have shown that ActD liposomes act synergistically with TRAIL liposomes in inducing apoptosis in the A-549 NSCLC cell line compared with treatment using ActD liposomes or TRAIL liposomes alone. The enhanced apoptosis is due to upregulation of DR5 induced by ActD liposomes in A-549 cells. These studies provide a foundation for the development of combined treatment regimens that would enhance the apoptotic response to TRAIL in cancer patients.

\section{Acknowledgments}

This work was supported by the National Basic Research Program of China (973 Program, 2007CB935800) and National Science and Technology Major Project 2009ZX09310-006. We thank Shanghai Qiaer Biotechnology Co., Ltd for supplying us with the TRAIL, and thank Dr Jing Sun for her help. 


\section{Disclosure}

The authors report no conflicts of interest in this work.

\section{References}

1. Ramalingam S, Belani C. Systemic chemotherapy for advanced non-small cell lung cancer: recent advances and future directions. Oncologist. 2008;13:5-13.

2. Dempkea WCM, Sutob T, Reckc M. Targeted therapies for non-small cell lung cancer. Lung Cancer. 2010;67:257-274.

3. Maemondo M, Inoue A, Inoue K, et al. Gefitinib or chemotherapy for non-small-cell lung cancer with mutated EGFR. $N$ Engl J Med. 2010;362:2380-2388.

4. Berger W, Setinek U, Hollaus $P$, et al. Multidrug resistance markers $\mathrm{P}$-glycoprotein, multidrug resistance protein 1 , and lung resistance protein in non-small cell lung cancer: prognostic implications. $J$ Cancer Res Clin Oncol. 2005;131:355-363.

5. Singh TR, Shankar S, Chen X, et al. Synergistic interactions of chemotherapeutic drugs and tumor necrosis factor-related apoptosis-inducing ligand/apo-2 ligand on apoptosis and on regression of breast carcinoma in vivo. Cancer Res. 2003;63:5390-5400.

6. Kruyt FAE. TRAIL and cancer therapy. Cancer Lett. 2008;263:14-25.

7. You M, Savaraj N, Wangpaichitr M, et al. The combination of ADIPEG20 and TRAIL effectively increases cell death in melanoma cell lines. Biochem Biophys Res Commun. 2010;394:760-766.

8. Kelley SK, Harris LA, Xie D, et al. Preclinical studies to predict the disposition of Apo2L/tumor necrosis factor-related apoptosis-inducing ligand in humans: characterization of in vivo efficacy, pharmacokinetics, and safety. J Pharmacol Exp Ther. 2001;29:31-38.

9. Ashkenazi A, Pai RC, Fong S, et al. Safety and antitumor activity of recombinant soluble Apo2 ligand. J Clin Invest. 1999;104: $155-162$.

10. Stegehuis JH, de Wilt LH, de Vries EG, Groen HJ, de Jong S, Kruyt FA. TRAIL receptor targeting therapies for non-small cell lung cancer: Current status and perspectives. Drug Resist Updat. 2010;13:2-15.

11. Wang MJ, Liu S, Liu Y, et al. Actinomycin D enhances TRAIL-induced caspase-dependent and -independent apoptosis in SH-SY5Y neuroblastoma cells. Neurosci Res. 2007;59:40-46.

12. Matsuzaki H, Schmied BM, Ulrich A, et al. Combination of tumor necrosis factor-related apoptosis-inducing ligand (TRAIL) and actinomycin D induces apoptosis even in TRAIL-resistant human pancreatic cancer cells. Clin Cancer Res. 2001;7:407-414.

13. Fan Q-L, Zou W-Y, Song L-Y, et al. Synergistic antitumor activity of TRAIL combined with chemotherapeutic agents in A549 cell lines in vitro and in vivo. Cancer Chemother Pharmacol. 2005;55: 189-196.

14. Walczak H, Miller RE, Ariail K, et al. Tumoricidal activity of tumor necrosis factor-related apoptosis-inducing ligand in vivo. Nat Med. 1999;5:157-163.

15. Gabizon A, Catane R, Uziely B, et al. Prolonged circulation time and enhanced accumulation in malignant exudates of doxorubicin encapsulated in polyethylene-glycol coated liposomes. Cancer Res. 1994;54:987-992.

16. Guo L, Fan L, Pang Z, et al. TRAIL and doxorubicin combination enhances anti-glioblastoma effect based on passive tumor targeting of liposomes. J Control Release. 2011;154:93-102.

17. Guo L, Fan L, Ren J, et al. A novel combination of TRAIL and doxorubicin enhances antitumor effect based on passive tumor-targeting of liposomes. Nanotechnology. 2011;22:265105.

18. Hira A, Watanabe H, Maeda Y, et al. Role of P-glycoprotein in accumulation and cytotoxicity of amrubicin and amrubicinol in MDR1 genetransfected LLC-PK1 cells and human A549 lung adenocarcinoma cells. Biochem Pharmacol. 2008;75:973-980.

19. Bradford MM. A rapid and sensitive method for the quantitation of microgram quantities of protein utilizing the principle of protein-dye binding. Anal Biochem. 1976;72:248-254.
20. Sun SY, Yue P, Wu GS, et al. Mechanisms of apoptosis induced by the synthetic retinoid CD437 in human non-small cell lung carcinoma cells. Oncogene. 1999;18:2357-2365.

21. Kok S-H, Yeh C-C, Chen M-L, et al. Esculetin enhances TRAIL-induced apoptosis through DR5 upregulation in human oral cancer SAS cells. Oral Oncol. 2009;45:1067-1072.

22. Harashima $\mathrm{H}$, Kiwada H. Liposomal targeting and drug delivery: kinetic consideration. Adv Drug Deliver Rev. 1996;19:425-444.

23. Wu GS. TRAIL as a target in anti-cancer therapy. Cancer Lett. 2008; 285:1-5

24. Pan G, Ni J, Wei Y-F, et al. An antagonist decoy receptor and a death domain-containing receptor for TRAIL. Science. 1997;277: 815-818.

25. Safa AR, Day TW, Wu CH. Cellular FLICE-like inhibitory protein (C-FLIP): a novel target for cancer therapy. Curr Cancer Drug Targets. 2008;8:37-46.

26. Eggert A, Grotzer MA, Zuzak TJ, et al. Resistance to TRAIL-induced apoptosis in neuroblastoma cells correlates with a loss of caspase- 8 expression. Med Pediatr Oncol. 2000;35:603-607.

27. Kim EH, Yoon MJ, Kim SU, et al. Arsenic trioxide sensitizes human glioma cells, but not normal astrocytes, to TRAIL-induced apoptosis via CCAAT/enhancer- binding protein homologous protein-dependent DR5 up-regulation. Cancer Res. 2008;68:266-275.

28. Frese S, Frese-Schaper M, Andres A-C, et al. Cardiac glycosides initiate Apo2L/TRAIL-induced apoptosis in non-small cell lung cancer cells by up-regulation of death receptors 4 and 5. Cancer Res. 2006;66: 5867-5873.

29. Liu X, Yue P, Zhou Z, et al. Death receptor regulation and celecoxibinduced apoptosis in human lung cancer cells. J Natl Cancer Inst. 2004;96:1769-1780.

30. Nakata S, Yoshida T, Horinaka M, et al. Histone deacetylase inhibitors upregulate death receptor 5/TRAIL-R2 and sensitize apoptosis induced by TRAIL/APO2-L in human malignant tumor cells. Oncogene. 2004;23:6261-6271.

31. Guan B, Yue P, Lotan R, et al. Evidence that the human death receptor 4 is regulated by activator protein 1. Oncogene. 2002;21: 3121-3129.

32. Frese S, Pirnia F, Miescher D, et al. PG490-mediated sensitization of lung cancer cells to Apo2L/TRAIL induced apoptosis requires activation of ERK2. Oncogene. 2003;22:5427-5435.

33. Song JH, Kandasamy K, As K. ABT-737 induces expression of the death receptor 5 and sensitizes human cancer cells to TRAIL-induced apoptosis. J Biol Chem. 2008;283:25003-25013.

34. Guan B, Yue P, Clayman G, et al. Evidence that the death receptor DR4 is a DNA damage-inducible, p53-regulated gene. J Cell Physiol. 2001;188:98-105.

35. Gómez-Benitoa M, Martinez-Lorenzob MJ, Anela A, et al. Membrane expression of DR4, DR5 and caspase-8 levels, but not Mcl-1, determine sensitivity of human myeloma cells to Apo2L/TRAIL. Exp Cell Res. 2007;313:2378-2388.

36. Zhang Y, Zhang B. TRAIL resistance of breast cancer cells is associated with constitutive endocytosis of death receptors 4 and 5. Mol Cancer Res. 2008;6:1861-1871.

37. Zauli G, Pandolfi A, Gonelli A, et al. Tumor necrosis factor-related apoptosis-inducing ligand (TRAIL) sequentially upregulates nitric oxide and prostanoid production in primary human endothelial cells. Circ Res. 2003;92:732-740.

38. Secchiero P, Gonelli A, Carnevale E, et al. Evidence for a proangiogenic activity of TNF-related apoptosis-inducing ligand. Neoplasia. 2004;6: 364-373.

39. Ishida T, Kiwada H. Accelerated blood clearance (ABC) phenomenon upon repeated injection of PEGylated liposomes. Int J Pharm. 2008;354: $56-62$.

40. Nagane M, Pan G, Weddle JJ, et al. Increased death receptor 5 expression by chemotherapeutic agents in human gliomas causes synergistic cytotoxicity with tumor necrosis factor-related apoptosis-inducing ligand in vitro and in vivo. Cancer Res. 2000;60:847-853. 
41. Wang S, Ren W, Liu J, et al. TRAIL and doxorubicin combination induces proapoptotic and antiangiogenic effects in soft tissue sarcoma in vivo. Clin Cancer Res. 2010;16:2591-2604.

42. Jin H, Yang R, Ross J, et al. Cooperation of the agonistic DR5 antibody apomab with chemotherapy to inhibit orthotopic lung tumor growth and improve survival. Clin Cancer Res. 2008;14:7733-7740.
43. Duiker EW, Mom CH, De Jong S, et al. The clinical trail of TRAIL. Eur J Cancer. 2006;42:2233-2240.

44. Werner AB, de Vries E, Tait SWG, et al. Bcl-2 family member Bfl-1/A1 sequesters truncated bid to inhibit its collaboration with proapoptotic Bak or Bax. J Biol Chem. 2002;277:22781-22788.

\section{Publish your work in this journal}

The International Journal of Nanomedicine is an international, peerreviewed journal focusing on the application of nanotechnology in diagnostics, therapeutics, and drug delivery systems throughout the biomedical field. This journal is indexed on PubMed Central, MedLine, CAS, SciSearch $\AA$, Current Contents ${ }^{\circledR} /$ Clinical Medicine,
Journal Citation Reports/Science Edition, EMBase, Scopus and the Elsevier Bibliographic databases. The manuscript management system is completely online and includes a very quick and fair peer-review system, which is all easy to use. Visit http://www.dovepress.com/ testimonials.php to read real quotes from published authors. 